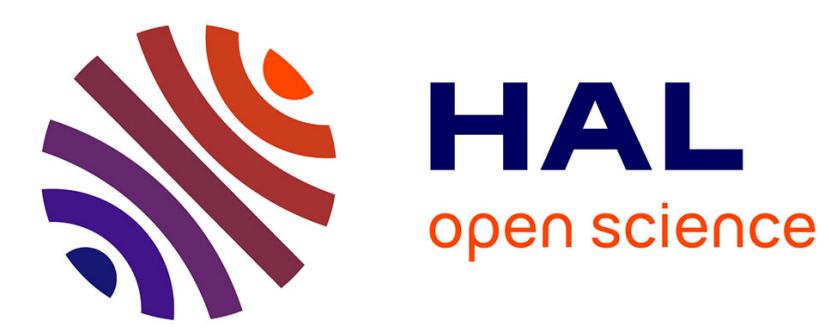

\title{
Weibull tail-distributions revisited: a new look at some tail estimators
}

Laurent Gardes, Stéphane Girard, Armelle Guillou

\section{To cite this version:}

Laurent Gardes, Stéphane Girard, Armelle Guillou. Weibull tail-distributions revisited: a new look at some tail estimators. Journal of Statistical Planning and Inference, 2011, 141 (1), pp.429-444. 10.1016/j.jspi.2010.06.018. hal-00340661v4

\section{HAL Id: hal-00340661 \\ https://hal.science/hal-00340661v4}

Submitted on 14 Jun 2010

HAL is a multi-disciplinary open access archive for the deposit and dissemination of scientific research documents, whether they are published or not. The documents may come from teaching and research institutions in France or abroad, or from public or private research centers.
L'archive ouverte pluridisciplinaire HAL, est destinée au dépôt et à la diffusion de documents scientifiques de niveau recherche, publiés ou non, émanant des établissements d'enseignement et de recherche français ou étrangers, des laboratoires publics ou privés. 


\title{
Weibull tail-distributions revisited: a new look at some tail estimators
}

\author{
Laurent Gardes $^{(1)}$, Stéphane Girard ${ }^{(1)} \&$ Armelle Guillou ${ }^{(2)}$ \\ (1) Team Mistis, INRIA Rhône-Alpes \& LJK, Inovallée, 655, av. de l'Europe, Montbonnot, \\ 38334 Saint-Ismier cedex, France \\ (2) Université de Strasbourg \& CNRS, IRMA, UMR 7501, 7 rue René Descartes, \\ 67084 Strasbourg cedex, France
}

\begin{abstract}
In this paper, we propose to include Weibull tail-distributions in a more general family of distributions. In particular, the considered model also encompasses the whole Fréchet maximum domain of attraction as well as log-Weibull tail-distributions. The asymptotic normality of some tail estimators based on the log-spacings between the largest order statistics is established in a unified way within the considered family. This result permits to understand the similarity between most estimators of the Weibull tail-coefficient and the Hill estimator. Some different asymptotic properties, in terms of bias, rate of convergence, are also highlighted.
\end{abstract}

AMS Subject Classifications: 62G05, 62G20, $62 \mathrm{G} 30$.

Keywords: Weibull tail-distributions, extreme quantile, maximum domain of attraction, asymptotic normality.

\section{Motivations}

Weibull tail-distributions encompass a variety of light-tailed distributions, i.e. distributions in the Gumbel maximum domain of attraction, see [20] for further details. Weibull tail-distributions include for instance Weibull, Gaussian, gamma and logistic distributions. Let us recall that a cumulative distribution function $F$ has a Weibull tail if its associated survival function $\bar{F}=1-F$ satisfies the following property: There exists $\theta>0$ such that for all $\lambda>0$,

$$
\lim _{t \rightarrow \infty} \frac{\log \bar{F}(\lambda t)}{\log \bar{F}(t)}=\lambda^{1 / \theta} .
$$

The parameter $\theta$ is called the Weibull tail-coefficient. We refer to [7] for a general account on Weibull tail-distributions and to [6] for an application to the modeling of large claims in non-life insurance. Dedicated methods have been proposed to estimate the Weibull tail-coefficient since the relevant information is only contained in the extreme upper part of the sample denoted hereafter by $X_{1}, \ldots, X_{n}$. A first direction was investigated in [8] where an estimator based on the record values is proposed. Another family of approaches $[3,4,10,13]$ consists of using the $k_{n}$ upper order statistics $X_{n-k_{n}+1, n} \leq \ldots \leq X_{n, n}$ where $\left(k_{n}\right)$ is an intermediate sequence of integers $i . e$. such that

$$
\lim _{n \rightarrow \infty} k_{n}=\infty \text { and } \lim _{n \rightarrow \infty} k_{n} / n=0
$$


More specifically, most recent estimators are based on the log-spacings between the $k_{n}$ upper order statistics [7, 11, 22, 23, 25, 26, 27]. All these estimators are thus similar to the Hill statistics [34] defined as

$$
H_{n}\left(k_{n}\right)=\frac{1}{k_{n}-1} \sum_{i=1}^{k_{n}-1} \log \left(X_{n-i+1, n}\right)-\log \left(X_{n-k_{n}+1, n}\right) .
$$

As an example, all three estimators proposed in [22] are proportional to $H_{n}\left(k_{n}\right)$. This similarity may be surprising since $H_{n}\left(k_{n}\right)$ is dedicated to the estimation of the tail index $\gamma$ for heavy-tailed distribution i.e. such that

$$
\lim _{t \rightarrow \infty} \frac{\bar{F}(\lambda t)}{\bar{F}(t)}=\lambda^{-1 / \gamma},
$$

for all $\lambda>0$. This property characterizes distributions belonging to the Fréchet maximum domain of attraction and sometimes called Pareto-type distributions.

The main goal of this work is therefore to explain why statistics based on log-spacings could be efficient in estimating tail parameters of both Weibull-tail and Pareto-type distributions. To this end, we introduce a family of distributions, indexed by two parameters $\tau \in[0,1]$ and $\theta>0$, which includes these two type of distributions. The first parameter $\tau$ allows to represent a large panel of distribution tails ranging from Weibull-type tails $(\tau=0)$ to Pareto-type tails $(\tau=1)$. The second parameter $\theta$ is the parameter to be estimated. It coincides with the Weibull tail-coefficient when $\tau=0$ and with the tail index when $\tau=1$.

An estimator $\widehat{\theta}_{n}\left(k_{n}\right)$ of $\theta$ is then introduced for the new family of distributions and an estimator of extreme quantiles is derived. The asymptotic normality of these estimators is established in Section 3 in a unified way and illustrated on some simulated data in Section 4. Some concluding remarks are given in Section 5. Proofs are postponed to Section 6.

\section{Model and estimators}

\subsection{Definition and first properties}

Let us consider the family of survival distribution functions defined as

$\left(\mathbf{A}_{1}(\tau, \theta)\right) \bar{F}(x)=\exp \left(-K_{\tau}^{\leftarrow}(\log H(x))\right)$ for $x \geq x_{*}$ with $x_{*}>0$ and

- $K_{\tau}(x)=\int_{1}^{x} u^{\tau-1} d u$ where $\tau \in[0,1]$,

- $H$ an increasing function such that $H^{\leftarrow}(t)=\inf \{x, H(x) \geq t\}=t^{\theta} \ell(t)$, where $\theta>0$ and $\ell$ is a slowly varying function i.e. $\ell(\lambda x) / \ell(x) \rightarrow 1$ as $x \rightarrow \infty$ for all $\lambda \geq 1$.

The function $H^{\leftarrow}$ is the so-called generalized inverse of $H$. Note that $K_{\tau}^{\leftarrow}$ coincides with the classical inverse since $K_{\tau}$ is continuous. The expansion $H^{\leftarrow}(t)=t^{\theta} \ell(t)$ is equivalent to supposing that $H^{\leftarrow}$ is regularly varying at infinity with index $\theta$. This property is denoted by $H^{\leftarrow} \in \mathcal{R}_{\theta}$, see [9] for more details on regular variations theory. Let us first highlight that the tail heaviness of $\bar{F}$ is mainly driven by $\tau \in[0,1]$ and secondarily by $\theta>0$ :

Proposition 1 Let $\bar{F}_{\tau_{1}, \theta_{1}}$ and $\bar{F}_{\tau_{2}, \theta_{2}}$ be two survival distribution functions satisfying respectively $\left(\mathbf{A}_{1}\left(\tau_{1}, \theta_{1}\right)\right)$ and $\left(\mathbf{A}_{1}\left(\tau_{2}, \theta_{2}\right)\right)$.

(i) If $\tau_{1}<\tau_{2}$ then $\bar{F}_{\tau_{1}, \theta_{1}}(x) / \bar{F}_{\tau_{2}, \theta_{2}}(x) \rightarrow 0$ as $x \rightarrow \infty$ for all $\left(\theta_{1}, \theta_{2}\right) \in(0, \infty)^{2}$.

(ii) If $\tau_{1}=\tau_{2}=\tau$ and $\theta_{1}<\theta_{2}$ then $\bar{F}_{\tau, \theta_{1}}(x) / \bar{F}_{\tau, \theta_{2}}(x) \rightarrow 0$ as $x \rightarrow \infty$. 
Thus, the larger is $\tau$, the heavier is the tail. Let us consider the two extremal cases $\tau=0$ and $\tau=1$. Clearly, under $\left(\mathbf{A}_{1}(0, \theta)\right), \bar{F}(x)=\exp (-H(x))$ is the survival function of a Weibull-tail distribution, see (1). At the opposite, $\left(\mathbf{A}_{1}(1, \theta)\right)$ entails $\bar{F}(x)=e^{1} / H(x)=x^{-1 / \theta} \tilde{\ell}(x)$ where $\tilde{\ell}$ is a slowly varying function. As a consequence, $F$ belongs to the Fréchet maximum domain of attraction and $\theta$ coincides with the tail index. In view of the above remarks, intermediate values of $\tau \in(0,1)$ correspond to distribution tails lighter than Pareto tails but heavier than Weibull tails. Indeed, we have $\bar{F}(x)=\exp (-h(x))$ with $h(x) \sim((\tau / \theta) \log x)^{1 / \tau}$ and thus $h(x) / x^{\beta} \rightarrow 0$ for all $\beta>0$ while $h(x) / \log (x) \rightarrow \infty$ as $x \rightarrow \infty$, this property characterizing an "exponential type" distribution, see [32]. The next proposition provides a more precise characterization while examples are provided in Paragraph 2.2.

\section{Proposition 2}

(i) F verifies $\left(\mathbf{A}_{1}(0, \theta)\right)$ if and only if $F$ is a Weibull-tail distribution function with Weibull tail-coefficient $\theta$.

(ii) If $F$ verifies $\left(\mathbf{A}_{1}(\tau, \theta)\right), \tau \in[0,1)$ and if $H$ is twice differentiable then $F$ belongs to the Gumbel maximum domain of attraction.

(iii) F verifies $\left(\mathbf{A}_{1}(1, \theta)\right)$ if and only if $F$ is in the Fréchet maximum domain of attraction with tail-index $\theta$.

\subsection{Examples}

In view of Proposition 2(i), Gaussian, gamma, Weibull, Benktander II, logistic and extreme-value distributions all verify $\left(\mathbf{A}_{1}(0, \theta)\right)$ since they are examples of Weibull tail-distributions (see [23], Table 1). Examples of distributions verifying $\left(\mathbf{A}_{1}(\tau, \theta)\right)$ with $\tau \in(0,1)$ include some log-Weibull tail-distributions. Let us recall that a random variable $Y$ is distributed from a log-Weibull taildistribution if $\log (Y)$ follows a Weibull tail-distribution.

Proposition 3 Suppose that $F$ verifies $\left(\mathbf{A}_{1}(0, \theta)\right)$ with $\theta \in(0,1]$. If, moreover, the slowly-varying function $\ell$ is differentiable and $\ell(t) \rightarrow \ell_{\infty}>0$ as $t \rightarrow \infty$ then $F(\log$.$) verifies \left(\mathbf{A}_{1}\left(\theta, \theta \ell_{\infty}\right)\right)$.

As an example, the standard log-normal distribution can be looked at as a log-Weibull taildistribution and thus verifies $\left(\mathbf{A}_{1}(1 / 2, \sqrt{2} / 2)\right)$. Similarly, the gamma distribution verifies $\left(\mathbf{A}_{1}(0,1)\right)$ and the log-gamma distribution belongs to the Fréchet maximum domain of attraction, see for instance [16], Table 3.4.2. Finally, other examples of distributions satisfying $\left(\mathbf{A}_{1}(1, \theta)\right)$ can be found in the above mentioned table.

\subsection{Definition of the estimators}

Denoting by $\left(k_{n}\right)$ an intermediate sequence of integers (see (2)), the following estimator of $\theta$ is considered:

$$
\widehat{\theta}_{n}\left(k_{n}\right)=\frac{1}{\mu_{1, \tau}\left(\log \left(n / k_{n}\right)\right)} \frac{1}{k_{n}-1} \sum_{i=1}^{k_{n}-1}\left(\log \left(X_{n-i+1, n}\right)-\log \left(X_{n-k_{n}+1, n}\right)\right),
$$

with, for all $t>0$ and $q \in \mathbb{N} \backslash\{0\}$,

$$
\mu_{q, \tau}(t)=\int_{0}^{\infty}\left(K_{\tau}(x+t)-K_{\tau}(t)\right)^{q} \mathrm{e}^{-x} d x .
$$


A crucial point is that the estimator (4) essentially consists in averaging the log-spacings between the upper-order statistics. Even more strongly, $\widehat{\theta}_{n}\left(k_{n}\right)$ only differs from the Hill statistics $(3)$ by a non-random normalizing sequence: $\widehat{\theta}_{n}\left(k_{n}\right)=H_{n}\left(k_{n}\right) / \mu_{1, \tau}\left(\log \left(n / k_{n}\right)\right)$. This similarity can be intuitively understood by studying the log-spacing between two quantiles $x_{u}$ and $x_{v}$ of $\bar{F}$, with $0<u<v \leq 1$. Under $\left(\mathbf{A}_{1}(\tau, \theta)\right)$ we have

$$
\log x_{u}-\log x_{v}=\theta\left(K_{\tau}(-\log u)-K_{\tau}(-\log v)\right)+\log \left(\frac{\ell\left(\exp K_{\tau}(-\log u)\right)}{\ell\left(\exp K_{\tau}(-\log v)\right)}\right) .
$$

Now, since $\ell$ is a slowly-varying function, if the orders $u$ and $v$ of the quantiles are small enough, the second term can be neglected in the right-hand side of (5) to obtain

$$
\log x_{u}-\log x_{v} \simeq \theta\left(K_{\tau}(-\log u)-K_{\tau}(-\log v)\right),
$$

which shows that log-spacings are approximately proportional to $\theta$. Since this key property holds for all $\tau \in[0,1]$, it is thus shared by Pareto-type, Weibull tail and $\log$-Weibull tail-distributions. Note that this property can be checked graphically on a sample by drawing a quantile-quantile plot. It consists in plotting the pairs $\left(K_{\tau}(\log (n / i)), \log \left(X_{n-i+1, n}\right)\right)$ for $i=1, \ldots, k_{n}$. From (6), the graph should be approximately linear. Following the same ideas, an estimator of the extreme quantile $x_{p_{n}}$ can be deduced from (4) by:

$$
\widehat{x}_{p_{n}}=X_{n-k_{n}+1, n} \exp \left(\widehat{\theta}_{n}\left(k_{n}\right)\left(K_{\tau}\left(\log \left(1 / p_{n}\right)\right)-K_{\tau}\left(\log \left(n / k_{n}\right)\right)\right)\right) .
$$

Recall that an extreme quantile $x_{p_{n}}$ of order $p_{n}$ is defined by $x_{p_{n}}=\bar{F}^{\leftarrow}\left(p_{n}\right)$ with $p_{n} \rightarrow 0$ as $n \rightarrow \infty$. For instance, if $n p_{n} \rightarrow 0$ then $x_{p_{n}}$ is larger than the maximum observation $X_{n, n}$ of the sample (with probability tending to one). This requires to extrapolate sample results to areas where no data are observed and occurs in reliability [14], hydrology [36], finance [16],...

\section{$3 \quad$ Asymptotic properties}

We show in the next paragraph that the asymptotic normality of $\widehat{\theta}_{n}\left(k_{n}\right)$ and $\widehat{x}_{p_{n}}$ can be established for all $\tau \in[0,1]$ in a unified way. In this sense, the asymptotic behavior of these estimators is more a consequence of the log-spacings property than of a tail behavior (which can be exponential as well as polynomial). Paragraphs 3.2 and 3.3 illustrate our general result on the two extremal cases $\tau=0$ and $\tau=1$.

\subsection{Main results}

To establish the asymptotic normality of $\widehat{\theta}_{n}\left(k_{n}\right)$, a second-order condition on $\ell$ is necessary:

$\left(\mathbf{A}_{2}(\rho)\right)$ There exist $\rho<0$ and $b(x) \rightarrow 0$ such that uniformly locally on $\lambda \geq \lambda_{0}>0$

$$
\log \left(\frac{\ell(\lambda x)}{\ell(x)}\right) \sim b(x) K_{\rho}(\lambda), \text { when } x \rightarrow \infty .
$$

It can be shown that necessarily $|b| \in \mathcal{R}_{\rho}$ (see [24]). The second order parameter $\rho<0$ tunes the rate of convergence of $\ell(\lambda x) / \ell(x)$ to 1 . The closer is $\rho$ to 0 , the slower is the convergence. Condition $\left(\mathbf{A}_{2}(\rho)\right)$ is the cornerstone in all the proofs of asymptotic normality for extreme value estimators. It is used in $[5,33,34]$ to prove the asymptotic normality of several estimators of the extreme value index. 
Theorem 1 Suppose that $\left(\mathbf{A}_{1}(\tau, \theta)\right)$ and $\left(\mathbf{A}_{2}(\rho)\right)$ hold. Let $\left(k_{n}\right)$ be an intermediate sequence such that

$$
\sqrt{k_{n}} b\left(\exp K_{\tau}\left(\log \left(n / k_{n}\right)\right)\right) \rightarrow \lambda .
$$

Then, introducing $a_{\tau, \rho}=1$ if $\tau \in[0,1)$ and $a_{1, \rho}=1 /(1-\rho)$, we have

$$
\sqrt{k_{n}}\left(\widehat{\theta}_{n}\left(k_{n}\right)-\theta-a_{\tau, \rho} b\left(\exp K_{\tau}\left(\log \left(n / k_{n}\right)\right)\right)\right) \stackrel{d}{\longrightarrow} \mathcal{N}\left(0, \theta^{2}\right) .
$$

It appears that the asymptotic variance of $\widehat{\theta}_{n}\left(k_{n}\right)$ given by $\mathcal{A V}=\theta^{2} / k_{n}$ is independent of $\tau$. In particular, it remains constant whatever the maximum domain of attraction of $F$. The asymptotic squared bias is given by $\mathcal{A S B}(\tau, \rho)=a_{\tau, \rho}^{2} b^{2}\left(\exp K_{\tau}\left(\log \left(n / k_{n}\right)\right)\right)$. If $b^{2}$ is ultimately decreasing, then $\mathcal{A S B}$ is a decreasing function of $\tau \in[0,1)$ with a jump at $\tau=1$. These remarks are illustrated on simulated data in Section 4. The next result allows us to establish the rate of convergence of $\widehat{\theta}_{n}\left(k_{n}\right)$ to $\theta$ in $(9)$.

Proposition 4 Condition (8) with $\lambda \neq 0$ implies $\log \left(k_{n}\right)=-2 \rho a_{\tau, 2 \rho} K_{\tau}(\log n)(1+o(1))$.

The rate of convergence is thus of order $\exp \left(-\rho a_{\tau, 2 \rho} K_{\tau}(\log n)(1+o(1))\right)$. A geometrical rate of convergence is obtained only in the Fréchet maximum domain of attraction, $\tau=1$ yields $\sqrt{k_{n}}=n^{-\rho /(1-2 \rho)+o(1)}$ which is consistent with the conclusions of [31]. Weibull tail-distributions give rise to logarithmic rates of convergence, $\tau=0$ yields $\sqrt{k_{n}}=(\log n)^{-\rho+o(1)}$ which is consistent with the results of [22]. More generally, the heavier is the tail, the better the rate of convergence is. The next result provides an extension of Statement 1 in [2], which was initially proved only for Weibull tail-distributions $(\tau=0)$.

Proposition 5 Suppose condition (8) holds with $\lambda \neq 0$. If $\tau \in[0,1 / 2]$ then

$$
\mathcal{A S B}(\tau, \rho)=c_{\tau, \rho} b^{2}\left(\exp K_{\tau}(\log n)\right)(1+o(1)),
$$

where $c_{\tau, \rho}=1$ if $\tau \in[0,1 / 2)$ and $c_{1 / 2, \rho}=\exp \left(8 \rho^{2}\right)$.

It follows that, when $\tau \in[0,1 / 2]$, the first order of the asymptotic bias is asymptotically independent of $k_{n}$. As a consequence, the asymptotic mean-squared error defined as $\mathcal{A S B}(\tau, \rho)+\mathcal{A V}$ is eventually decreasing with respect to $k_{n}$. This remark, already made in [2] in the particular case $\tau=0$, is only of theoretical interest. Indeed, in finite sample situations, condition (8) does not hold and the empirical mean-squared error is a convex function of $k_{n}$, see for instance [22]. Now, the asymptotic normality of the extreme quantile estimator (7) can be deduced from Theorem 1:

Theorem 2 Suppose the assumptions of Theorem 1 hold with $\lambda=0$. If, moreover,

$$
\left(\log \left(n / k_{n}\right)\right)^{1-\tau}\left(K_{\tau}\left(\log \left(1 / p_{n}\right)\right)-K_{\tau}\left(\log \left(n / k_{n}\right)\right)\right) \rightarrow \infty
$$

then,

$$
\frac{\sqrt{k_{n}}}{K_{\tau}\left(\log \left(1 / p_{n}\right)\right)-K_{\tau}\left(\log \left(n / k_{n}\right)\right)}\left(\frac{\widehat{x}_{p_{n}}}{x_{p_{n}}}-1\right) \stackrel{d}{\longrightarrow} \mathcal{N}\left(0, \theta^{2}\right) .
$$

Let us now focus on the two particular cases $\tau=0$ (Weibull tail-distributions) and $\tau=1$ (Fréchet maximum domain of attraction). 


\subsection{Application to Weibull tail-distributions}

If $\tau=0$, the estimator (4) coincides with $\widehat{\theta}_{n}^{(1)}$ introduced in [22], and

$$
\widehat{x}_{p_{n}}=X_{n-k_{n}+1, n}\left(\frac{\log \left(1 / p_{n}\right)}{\log \left(n / k_{n}\right)}\right)^{\widehat{\theta}_{n}\left(k_{n}\right)}
$$

is the estimator proposed in [21]. As a consequence of Theorem 1 and Theorem 2, we obtain:

Corollary 1 Suppose that $\left(\mathbf{A}_{1}(0, \theta)\right)$ and $\left(\mathbf{A}_{2}(\rho)\right)$ hold. Let $\left(k_{n}\right)$ be an intermediate sequence such that $\sqrt{k_{n}} b\left(\log \left(n / k_{n}\right)\right) \rightarrow 0$. Then,

$$
\sqrt{k_{n}}\left(\widehat{\theta}_{n}\left(k_{n}\right)-\theta\right) \stackrel{d}{\longrightarrow} \mathcal{N}\left(0, \theta^{2}\right) .
$$

If, moreover

$$
\log \left(n / k_{n}\right)\left(\log \log \left(1 / p_{n}\right)-\log \log \left(n / k_{n}\right)\right) \rightarrow \infty
$$

then,

$$
\frac{\sqrt{k_{n}}}{\log \log \left(1 / p_{n}\right)-\log \log \left(n / k_{n}\right)}\left(\frac{\widehat{x}_{p_{n}}}{x_{p_{n}}}-1\right) \stackrel{d}{\longrightarrow} \mathcal{N}\left(0, \theta^{2}\right) .
$$

This result is very similar to Corollary 3.1 in [22] except that condition (11) is weaker than the one used in the above mentioned paper. Let us also note that estimators $\widehat{\theta}_{n}^{(2)}$ and $\widehat{\theta}_{n}^{(3)}$ in [22] can be respectively deduced from $\widehat{\theta}_{n}$ by approximating $\mu_{1,0}$ by a Riemann's sum or using the first order approximation $\mu_{1,0}(t) \sim 1 / t$ as $t \rightarrow \infty$ given in Lemma $2(\mathrm{i})$.

\subsection{Application to the Fréchet maximum domain of attraction}

Letting $\tau=1$ and remarking that $\mu_{q, 1}(t)=q$ ! for all $t>0$ and $q \in \mathbb{N} \backslash\{0\}$, the estimator (4) coincides with (3) which is the Hill estimator [34] of the tail index. Besides,

$$
\widehat{x}_{p_{n}}=X_{n-k_{n}+1, n}\left(\frac{k_{n}}{n p_{n}}\right)^{\widehat{\theta}_{n}\left(k_{n}\right)}
$$

is the Weissman estimator [37]. A straightforward application of the above theorems gives back the classical results:

Corollary 2 Suppose that $\left(\mathbf{A}_{1}(1, \theta)\right)$ and $\left(\mathbf{A}_{2}(\rho)\right)$ hold. Let $\left(k_{n}\right)$ be an intermediate sequence such that $\sqrt{k_{n}} b\left(n / k_{n}\right) \rightarrow 0$. Then,

$$
\sqrt{k_{n}}\left(\widehat{\theta}_{n}\left(k_{n}\right)-\theta\right) \stackrel{d}{\longrightarrow} \mathcal{N}\left(0, \theta^{2}\right) .
$$

If, moreover $k_{n} /\left(n p_{n}\right) \rightarrow \infty$ then,

$$
\frac{\sqrt{k_{n}}}{\log \left(k_{n} /\left(n p_{n}\right)\right)}\left(\frac{\widehat{x}_{p_{n}}}{x_{p_{n}}}-1\right) \stackrel{d}{\longrightarrow} \mathcal{N}\left(0, \theta^{2}\right) .
$$




\section{Illustration on simulations}

The section is dedicated to the illustration of the conclusions drawn from Theorem 1 on simulated data. To this end, we consider a cumulative distribution function $F_{\theta, \tau, \rho}$ verifying $\left(\mathbf{A}_{1}(\tau, \theta)\right)$ and $\left(\mathbf{A}_{2}(\rho)\right)$ with $\theta=1 / 2, \tau \in\{0,1 / 2,1\}$ and $\rho \in\{-1 / 2,-1 / 4\}$. More specifically, the slowly-varying function is given by

$$
\ell(x)=1-\frac{\theta}{\rho}(1+x)^{\rho}\left(1+\frac{1}{x}\right)^{\theta} .
$$

Following Proposition 2(i), it appears that the case $\tau=0$ corresponds to a Weibull tail-distribution (with Weibull tail-coefficient $1 / 2$ ) similar to a Gaussian distribution. When $\tau=1 / 2$, in view of Paragraph 2.1, $\bar{F}(x)=\exp \left\{-(\log x)^{2}(1+o(1))\right\}$, the distribution has a tail behavior similar to the log-normal distribution. Finally, Proposition 2(iii) shows that, when $\tau=1$, the distribution belongs to the Fréchet maximum domain of attraction with tail-index 1/2.

For each considered combination of $\tau$ and $\rho, N=500$ samples $\left(\mathcal{X}_{n, j}\right)_{j=1, \ldots, N}$ of size $n=500$ were simulated from $F_{1 / 2, \tau, \rho}$. On each sample $\left(\mathcal{X}_{n, j}\right)$, the estimate $\hat{\theta}_{n, j}$ is computed for $k=2, \ldots, 250$, the associated empirical squared bias $\mathcal{E} \mathcal{S B}$ and empirical variance $\mathcal{E} V$ plots are built by plotting the pairs $\left(k,\left(\bar{\theta}_{n}^{(1)}(k)-\theta\right)^{2}\right)$ and $\left(k, \bar{\theta}_{n}^{(2)}(k)-\left(\bar{\theta}_{n}^{(1)}(k)\right)^{2}\right)$ where for $i \in\{1,2\}$,

$$
\bar{\theta}_{n}^{(i)}(k)=\frac{1}{N} \sum_{j=1}^{N}\left(\hat{\theta}_{n, j}(k)\right)^{i} .
$$

The empirical squared bias and the empirical variance are depicted on Figure 1 and Figure 2 respectively. Both graphs are represented on the same scale for the sake of comparison. As expected, the squared bias, for a fixed value of $k$, is an increasing function of $\rho$ and a decreasing function of $\tau$. At the opposite, the variance seems to be independent of $\rho$ and is not much dependent of $\tau$.

\section{Concluding remarks}

As illustrated in the previous sections, the model $\left(\mathbf{A}_{1}(\tau, \theta)\right)$ provides a new tool for the analysis of tail estimators based on log-spacings. It allows us to encompass Weibull tail-distributions in a more general framework and thus to explain why their dedicated tail estimators are very similar to Hill or Weissman statistics. The next step would be to estimate the parameter $\tau$. For instance, one can consider the following estimator based on the log-spacing between two Hill statistics

$$
\widehat{\tau}_{n}=1+\frac{\log H_{n}\left(k_{n}^{\prime}\right)-\log H_{n}\left(k_{n}\right)}{\log \log \left(n / k_{n}^{\prime}\right)-\log \log \left(n / k_{n}\right)},
$$

where $\left(k_{n}\right)$ and $\left(k_{n}^{\prime}\right)$ are two intermediate sequences such that

$$
\liminf _{n \rightarrow \infty} \frac{\log \left(n / k_{n}^{\prime}\right)}{\log \left(n / k_{n}\right)}>1
$$

Let us note that

$$
\begin{aligned}
\left(\log \log \left(n / k_{n}^{\prime}\right)-\log \log \left(n / k_{n}\right)\right)\left(\widehat{\tau}_{n}-\tau\right) & =\log \left(\widehat{\theta}_{n}\left(k_{n}^{\prime}\right) / \theta\right)-\log \left(\widehat{\theta}_{n}\left(k_{n}\right) / \theta\right) \\
& +\log \left(\frac{\mu_{1, \tau}\left(\log \left(n / k_{n}^{\prime}\right)\right)}{\log ^{\tau-1}\left(n / k_{n}^{\prime}\right)}\right)-\log \left(\frac{\mu_{1, \tau}\left(\log \left(n / k_{n}\right)\right)}{\log ^{\tau-1}\left(n / k_{n}\right)}\right) .
\end{aligned}
$$


This implies that the consistency of $\widehat{\tau}_{n}$ is a simple consequence of Theorem 1 and Lemma 2(i) whereas the asymptotic distribution is much more difficult to handle as it requires the joint distribution of $\widehat{\theta}_{n}\left(k_{n}^{\prime}\right)$ and $\widehat{\theta}_{n}\left(k_{n}\right)$. Also in practice, the choice of the parameters $k_{n}$ and $k_{n}^{\prime}$ is an open question. These two points are currently under investigation.

Other extensions are possible, among others bias correction based on the estimation of the secondorder parameter $[28,29]$. To this end, an exponential regression model for these tail distributions extending $[5,11,12,18]$ would be of interest. We also plan to adapt our results to the case $\tau>1$ and to investigate the possible links with super-heavy tails [19]. Finally, this work could be further extended to random variables $Y=\psi(X)$ where $X$ has a parent distribution satisfying $\left(\mathbf{A}_{1}(\tau, \theta)\right)$. For instance, choosing $\psi(x)=x^{*}-1 / x$ would allow to consider distributions (with finite endpoint $x^{*}$ ) in the Weibull maximum domain of attraction. This may help for including the negative Hill estimator (see for instance [17] or [30], paragraph 3.6.2) in our framework.

\section{Proofs}

We first give some preliminary lemmas. Their proofs are postponed to the appendix.

\subsection{Preliminary lemmas}

The first lemma provides some uniform approximations based on $\left(\mathbf{A}_{1}(\tau, \theta)\right)$ and $\left(\mathbf{A}_{2}(\rho)\right)$.

Lemma 1 If $\left(\mathbf{A}_{1}(\tau, \theta)\right)$ and $\left(\mathbf{A}_{2}(\rho)\right)$ hold then

$$
\sup _{\lambda \geq 1}\left|\frac{\ell(\lambda x)}{\ell(x)}-1-b(x) K_{\rho}(\lambda)\right|=o(b(x)) \text {, when } x \rightarrow \infty .
$$

Let us define for all $q \in \mathbb{N} \backslash\{0\}, \tau \in[0,1]$ and $t>0, \sigma_{q, \tau}^{2}(t)=\mu_{2 q, \tau}(t)-\mu_{q, \tau}^{2}(t)$. The following lemma is of analytical nature. It provides first-order expansions which will be useful in the sequel.

Lemma 2 For all $q \in \mathbb{N} \backslash\{0\}$ and $\tau \in[0,1]$, when $t \rightarrow \infty$ :

(i) $\mu_{q, \tau}(t) \sim q ! t^{(\tau-1) q}$,

(ii) $\sigma_{q, \tau}^{2}(t) / \mu_{q, \tau}^{2}(t) \rightarrow(2 q) ! /(q !)^{2}-1$,

(iii) $\mu_{1, \tau}^{\prime}(t) / \mu_{1, \tau}(t) \rightarrow 0$.

The next lemma presents an expansion of $\widehat{\theta}_{n}\left(k_{n}\right)$.

Lemma 3 Let $\left(k_{n}\right)$ be an intermediate sequence. Then, under $\left(\mathbf{A}_{1}(\tau, \theta)\right)$, the following expansions hold:

$$
\widehat{\theta}_{n}\left(k_{n}\right)=\frac{1}{\mu_{1, \tau}\left(\log \left(n / k_{n}\right)\right)}\left(\theta \theta_{n, 1}^{(1)}\left(E_{n-k_{n}+1, n}\right)+\theta_{n, 2}\left(E_{n-k_{n}+1, n}\right)\right),
$$

with, for all $q \in \mathbb{N} \backslash\{0\}$,

$$
\begin{aligned}
\theta_{n, 1}^{(q)}(t) & =\frac{1}{k_{n}-1} \sum_{i=1}^{k_{n}-1}\left(K_{\tau}\left(F_{i}+t\right)-K_{\tau}(t)\right)^{q} \\
\theta_{n, 2}(t) & =\frac{1}{k_{n}-1} \sum_{i=1}^{k_{n}-1} \log \left(\frac{\ell\left(\exp K_{\tau}\left(F_{i}+t\right)\right)}{\ell\left(\exp K_{\tau}(t)\right)}\right)
\end{aligned}
$$

and where $E_{n-k_{n}+1, n}$ is the $\left(n-k_{n}+1\right)$ th order statistic associated to $n$ independent standard exponential variables and $\left\{F_{1}, \ldots, F_{k_{n}-1}\right\}$ are independent standard exponential variables and independent from $E_{n-k_{n}+1, n}$. 
The asymptotic behavior of the $\left(n-k_{n}+1\right)$ th standard exponential order statistic is described in the following lemma.

Lemma 4 Let $\left(k_{n}\right)$ be an intermediate sequence. Then, for all differentiable function $g$, we have

$$
\sqrt{k_{n}}\left(g\left(E_{n-k_{n}+1, n}\right)-g\left(\log \left(n / k_{n}\right)\right)\right)=O_{\mathbb{P}}(1) g^{\prime}\left(\log \left(n / k_{n}\right)\left(1+o_{\mathbb{P}}(1)\right)\right) .
$$

The next two lemmas provide the key results for establishing the asymptotic distribution of $\widehat{\theta}_{n}\left(k_{n}\right)$. They describe the asymptotic behavior of the random terms appearing in Lemma 3.

Lemma 5 Let $\left(k_{n}\right)$ be an intermediate sequence. Then, for all $q \in \mathbb{N} \backslash\{0\}$,

$$
\frac{\sqrt{k_{n}}}{\sigma_{q, \tau}\left(E_{n-k_{n}+1, n}\right)}\left(\theta_{n, 1}^{(q)}\left(E_{n-k_{n}+1, n}\right)-\mu_{q, \tau}\left(E_{n-k_{n}+1, n}\right)\right) \stackrel{d}{\longrightarrow} \mathcal{N}(0,1)
$$

Lemma 6 Suppose that $\left(\mathbf{A}_{1}(\tau, \theta)\right)$ and $\left(\mathbf{A}_{2}(\rho)\right)$ hold. Let $\left(k_{n}\right)$ be an intermediate sequence. Then,

$$
\theta_{n, 2}\left(E_{n-k_{n}+1, n}\right)=b\left(\exp K_{\tau}\left(E_{n-k_{n}+1, n}\right)\right) \theta_{n, 3}\left(E_{n-k_{n}+1, n}\right)\left(1+o_{\mathbb{P}}(1)\right),
$$

where

$$
\left|\theta_{n, 3}\left(E_{n-k_{n}+1, n}\right)-\theta_{n, 1}^{(1)}\left(E_{n-k_{n}+1, n}\right)\right| \leq-\frac{\rho}{2} \theta_{n, 1}^{(2)}\left(E_{n-k_{n}+1, n}\right) .
$$

Moreover, if $\tau=1$, then $\theta_{n, 3}\left(E_{n-k_{n}+1, n}\right) \stackrel{P}{\longrightarrow} 1 /(1-\rho)$.

\subsection{Proofs of the main results}

Proof of Proposition 1 - Assumptions $\left(\mathbf{A}_{1}\left(\tau_{1}, \theta_{1}\right)\right)$ and $\left(\mathbf{A}_{1}\left(\tau_{2}, \theta_{2}\right)\right)$ entail

$$
\frac{\bar{F}_{\tau_{1}, \theta_{1}}(x)}{\bar{F}_{\tau_{2}, \theta_{2}}(x)}=\exp \left[-K_{\tau_{1}}^{\leftarrow}\left(\log H_{1}(x)\right)\left(1-\frac{K_{\tau_{2}}^{\leftarrow}\left(\log H_{2}(x)\right)}{K_{\tau_{1}}^{\leftarrow}\left(\log H_{1}(x)\right)}\right)\right]
$$

where $H_{1} \in \mathcal{R}_{1 / \theta_{1}}$ and $H_{2} \in \mathcal{R}_{1 / \theta_{2}}$. As a consequence, for all $q \in\{1,2\}, \log H_{q}(x) \sim \log (x) / \theta_{q}$ when $x \rightarrow \infty$, see [9], Proposition 1.3.6. Let us first prove (i): $0<\tau_{1}<\tau_{2}$ implies

$$
K_{\tau_{q}}^{\leftarrow}\left(\log H_{q}(x)\right) \sim\left(\tau_{q} / \theta_{q}\right)^{1 / \tau_{q}}(\log x)^{1 / \tau_{q}} \rightarrow \infty,
$$

and thus

$$
\frac{K_{\tau_{2}}^{\leftarrow}\left(\log H_{2}(x)\right)}{K_{\tau_{1}}^{\leftarrow}\left(\log H_{1}(x)\right)} \sim \frac{\left(\tau_{2} / \theta_{2}\right)^{1 / \tau_{2}}}{\left(\tau_{1} / \theta_{1}\right)^{1 / \tau_{1}}}(\log x)^{1 / \tau_{2}-1 / \tau_{1}} \rightarrow 0
$$

Collecting (12), (13) and (14) gives the result: $\bar{F}_{\tau_{1}, \theta_{1}}(x) / \bar{F}_{\tau_{2}, \theta_{2}}(x) \rightarrow 0$ as $x \rightarrow \infty$. Similarly, if $\tau_{1}=0$, then

$$
\frac{K_{\tau_{2}}^{\leftarrow}\left(\log H_{2}(x)\right)}{K_{0}^{\leftarrow}\left(\log H_{1}(x)\right)} \sim \frac{\left(\tau_{2} / \theta_{2}\right)^{1 / \tau_{2}}}{H_{1}(x)}(\log x)^{1 / \tau_{2}} \rightarrow 0
$$

which concludes the first part of the proof. Let us now focus on (ii) and suppose $\theta_{1}<\theta_{2}$. If $\tau>0$ then

$$
\frac{K_{\tau}^{\leftarrow}\left(\log H_{2}(x)\right)}{K_{\tau}^{\leftarrow}\left(\log H_{1}(x)\right)} \rightarrow\left(\frac{\theta_{1}}{\theta_{2}}\right)^{1 / \tau}<1
$$

as $x \rightarrow \infty$, while, if $\tau=0$,

$$
\frac{K_{0}^{\leftarrow}\left(\log H_{2}(x)\right)}{K_{0}^{\leftarrow}\left(\log H_{1}(x)\right)}=\frac{H_{2}(x)}{H_{1}(x)} \rightarrow 0
$$

as $x \rightarrow \infty$. In both cases, for $x$ large enough,

$$
1-\frac{K_{\tau}^{\leftarrow}\left(\log H_{2}(x)\right)}{K_{\tau}^{\leftarrow}\left(\log H_{1}(x)\right)}>0,
$$

and collecting (12), (13) and (15) concludes the proof: $\bar{F}_{\tau, \theta_{1}}(x) / \bar{F}_{\tau, \theta_{2}}(x) \rightarrow 0$ as $x \rightarrow \infty$. 
Proof of Proposition 2 - Proofs of (i) and (iii) are straightforward consequences of Paragraph 2.1. Let us focus on (ii). In view of the characterization (3.35) in [16] of the Gumbel maximum domain of attraction, it is sufficient to prove that there exists a positive function $a$, differentiable with $a^{\prime}(t) \rightarrow 0$ as $t \rightarrow \infty$, such that

$$
\bar{F}(x)=\exp \left\{-\int_{x_{*}}^{x} \frac{d t}{a(t)}\right\}, x \geq x_{*} .
$$

Letting $a=1 /\left(K_{\tau}^{\leftarrow}(\log H)\right)^{\prime}$, it thus remains to prove that $a^{\prime}(t) \rightarrow 0$ as $t \rightarrow \infty$ for all $\tau \in[0,1)$. To this end, let us remark that

$$
\begin{aligned}
a^{\prime}(t) & =\frac{1}{K_{\tau}^{\leftarrow}(\log H(t))}\left(\tau-1+\left(1-\frac{H^{\prime \prime}(t) H(t)}{H^{\prime}(t)^{2}}\right)(1+\tau \log H(t))\right) \\
& =\frac{1}{K_{\tau}^{\leftarrow}(\log H(t))}(\tau-1+(\theta+o(1))(1+\tau \log H(t))),
\end{aligned}
$$

since $H^{\prime} \in \mathcal{R}_{1 / \theta-1}$ implies $H^{\prime \prime}(t) H(t) / H^{\prime}(t)^{2} \rightarrow 1-\theta$ as $t \rightarrow \infty$. Two cases arise:

- If $\tau \in(0,1)$ then $a^{\prime}(t) \sim \theta(\tau \log H(t))^{1-1 / \tau} \rightarrow 0$ as $t \rightarrow \infty$.

- Otherwise, when $\tau=0$, we have $a^{\prime}(t)=(\theta-1+o(1)) / H(t) \rightarrow 0$ as $t \rightarrow \infty$.

In both situations, the conclusion follows.

Proof of Proposition $\mathbf{3}$ - Let us suppose that $F$ verifies $\left(\mathbf{A}_{1}(0, \theta)\right)$ with $\theta \in(0,1]$. Then, introducing $W(x)=\exp K_{\theta}(H(\log x))$, we have $\bar{F}(\log x)=\exp \left(-K_{\theta}^{\leftarrow}(\log W(x))\right)$. It thus remains to prove that $W^{\leftarrow} \in \mathcal{R}_{\theta \ell_{\infty}}$. Simple calculations show that

$$
\begin{aligned}
W^{\leftarrow}(t) & =\exp \left\{H^{\leftarrow}\left(K_{\theta}^{\leftarrow}(\log t)\right)\right\} \\
& =\exp \left\{(1+\theta \log t) \ell\left(K_{\theta}^{\leftarrow}(\log t)\right)\right\} \\
& =\mathrm{e}^{\ell_{\infty}} t^{\theta \ell_{\infty}} \varphi(t),
\end{aligned}
$$

where we have defined $\varphi(t)=\psi(\log t)$ with $\psi(x)=\exp \left\{(1+\theta x)\left[\ell\left(K_{\theta}^{\leftarrow}(x)\right)-\ell_{\infty}\right]\right\}$. As a consequence,

$$
\begin{aligned}
t(\log \varphi(t))^{\prime} & =(\log \psi)^{\prime}(\log t) \\
& =\theta\left(\ell\left(K_{\theta}^{\leftarrow}(\log t)\right)-\ell_{\infty}\right)+K_{\theta}^{\leftarrow}(\log t) \ell^{\prime}\left(K_{\theta}^{\leftarrow}(\log t)\right) \\
& =o(1),
\end{aligned}
$$

since, from [9], p. 15, $u \ell^{\prime}(u) / \ell(u) \rightarrow 0$ as $u \rightarrow \infty$. Using again [9], p. 15, it follows that $\varphi$ is a slowly varying function. Thus, $W^{\leftarrow} \in \mathcal{R}_{\theta \ell_{\infty}}$ and $F(\log$.$) verifies \left(\mathbf{A}_{1}\left(\theta, \theta \ell_{\infty}\right)\right)$.

Proof of Theorem 1 - Lemma 5 states that for $q \in\{1,2\}$,

$$
\frac{\sqrt{k_{n}}}{\sigma_{q, \tau}\left(E_{n-k_{n}+1, n}\right)}\left(\theta_{n, 1}^{(q)}\left(E_{n-k_{n}+1, n}\right)-\mu_{q, \tau}\left(E_{n-k_{n}+1, n}\right)\right)=\xi_{n}^{(q)}
$$

where $\xi_{n}^{(q)} \stackrel{d}{\longrightarrow} \mathcal{N}(0,1)$. Then, by Lemma 3 ,

$$
\begin{aligned}
\sqrt{k_{n}}\left(\widehat{\theta}_{n}\left(k_{n}\right)-\theta-a_{\tau, \rho} b\left(\exp K_{\tau}\left(\log \left(n / k_{n}\right)\right)\right)\right. & =\sqrt{k_{n}} \theta\left(\frac{\mu_{1, \tau}\left(E_{n-k_{n}+1, n}\right)}{\mu_{1, \tau}\left(\log \left(n / k_{n}\right)\right)}-1\right)+\theta \frac{\sigma_{1, \tau}\left(E_{n-k_{n}+1, n}\right)}{\mu_{1, \tau}\left(\log \left(n / k_{n}\right)\right)} \xi_{n}^{(1)} \\
& +\sqrt{k_{n}}\left(\frac{\theta_{n, 2}\left(E_{n-k_{n}+1, n}\right)}{\mu_{1, \tau}\left(\log \left(n / k_{n}\right)\right)}-a_{\tau, \rho} b\left(\exp K_{\tau}\left(\log \left(n / k_{n}\right)\right)\right)\right. \\
& \stackrel{\text { def }}{=} T_{n}^{(1)}+T_{n}^{(2)}+T_{n}^{(3)},
\end{aligned}
$$


and the three terms are studied separately. First, applying Lemma 4 to $g=\mu_{1, \tau}$ yields

$$
T_{n}^{(1)}=O_{\mathbb{P}}(1) \frac{\mu_{1, \tau}^{\prime}\left(\log \left(n / k_{n}\right)\left(1+o_{\mathbb{P}}(1)\right)\right)}{\mu_{1, \tau}\left(\log \left(n / k_{n}\right)\right)}=o_{\mathbb{P}}(1),
$$

in view of Lemma 2(i, iii). Second,

$$
T_{n}^{(2)}=\frac{\sigma_{1, \tau}\left(E_{n-k_{n}+1, n}\right)}{\mu_{1, \tau}\left(E_{n-k_{n}+1, n}\right)}\left(1+\frac{T_{n}^{(1)}}{\theta \sqrt{k_{n}}}\right) \theta \xi_{n}^{(1)}=\frac{\sigma_{1, \tau}\left(E_{n-k_{n}+1, n}\right)}{\mu_{1, \tau}\left(E_{n-k_{n}+1, n}\right)} \theta \xi_{n}^{(1)}\left(1+o_{\mathbb{P}}(1)\right)
$$

and, from Lemma $2(\mathrm{ii}), \sigma_{1, \tau}\left(E_{n-k_{n}+1, n}\right) / \mu_{1, \tau}\left(E_{n-k_{n}+1, n}\right) \stackrel{P}{\longrightarrow} 1$. As a preliminary conclusion,

$$
T_{n}^{(2)}=\theta \xi_{n}^{(1)}\left(1+o_{\mathbb{P}}(1)\right) .
$$

From Lemma $6, T_{n}^{(3)}$ can be expanded as

$$
\begin{aligned}
T_{n}^{(3)} & =\sqrt{k_{n}} b\left(\exp K_{\tau}\left(\log \left(n / k_{n}\right)\right)\right)\left(\frac{b\left(\exp K_{\tau}\left(E_{n-k_{n}+1, n}\right)\right)}{b\left(\exp K_{\tau}\left(\log \left(n / k_{n}\right)\right)\right)} \frac{\theta_{n, 3}\left(E_{n-k_{n}+1, n}\right)}{\mu_{1, \tau}\left(\log \left(n / k_{n}\right)\right)}\left(1+o_{\mathbb{P}}(1)\right)-a_{\tau, \rho}\right) \\
& =\lambda\left(\frac{b\left(\exp K_{\tau}\left(E_{n-k_{n}+1, n}\right)\right)}{b\left(\exp K_{\tau}\left(\log \left(n / k_{n}\right)\right)\right)} \frac{\theta_{n, 3}\left(E_{n-k_{n}+1, n}\right)}{\mu_{1, \tau}\left(\log \left(n / k_{n}\right)\right)}\left(1+o_{\mathbb{P}}(1)\right)-a_{\tau, \rho}\right)(1+o(1)) .
\end{aligned}
$$

Introducing $T_{n}^{(3,1)}=K_{\tau}\left(E_{n-k_{n}+1, n}\right)-K_{\tau}\left(\log \left(n / k_{n}\right)\right)$ and applying Lemma 4 with $g=K_{\tau}$ yield

$$
\exp T_{n}^{(3,1)}=\exp \left(O_{\mathbb{P}}(1) \frac{\left(\log \left(n / k_{n}\right)\right)^{\tau-1}}{\sqrt{k_{n}}}\right) \stackrel{P}{\longrightarrow} 1,
$$

since $\tau \in[0,1]$. Therefore, $b$ being regularly varying,

$$
b\left(\exp K_{\tau}\left(E_{n-k_{n}+1, n}\right) / b\left(\exp K_{\tau}\left(\log \left(n / k_{n}\right)\right) \stackrel{P}{\longrightarrow} 1\right.\right.
$$

as well, and consequently

$$
\begin{aligned}
T_{n}^{(3)} & =\lambda\left(\frac{\theta_{n, 3}\left(E_{n-k_{n}+1, n}\right)}{\mu_{1, \tau}\left(\log \left(n / k_{n}\right)\right)}\left(1+o_{\mathbb{P}}(1)\right)-a_{\tau, \rho}\right)(1+o(1)) \\
& =\lambda\left(\frac{\theta_{n, 3}\left(E_{n-k_{n}+1, n}\right)}{\mu_{1, \tau}\left(E_{n-k_{n}+1, n}\right)}\left(1+\frac{T_{n}^{(1)}}{\theta \sqrt{k_{n}}}\right)\left(1+o_{\mathbb{P}}(1)\right)-a_{\tau, \rho}\right)(1+o(1)) \\
& =\lambda\left(\frac{\theta_{n, 3}\left(E_{n-k_{n}+1, n}\right)}{\mu_{1, \tau}\left(E_{n-k_{n}+1, n}\right)}\left(1+o_{\mathbb{P}}(1)\right)-a_{\tau, \rho}\right)(1+o(1)),
\end{aligned}
$$

from (17). Two situations occur. If $\tau=1$, then, in view of Lemma $6, \theta_{n, 3}\left(E_{n-k_{n}+1, n}\right) \stackrel{P}{\longrightarrow} a_{1, \rho}=$ $1 /(1-\rho), \mu_{1,1}\left(E_{n-k_{n}+1, n}\right)=1$ and thus $T_{n}^{(3)} \stackrel{P}{\longrightarrow} 0$. If $\tau \in[0,1), T_{n}^{(3)}$ can be rewritten as

$$
T_{n}^{(3)}=\lambda\left(\left(T_{n}^{(3,2)}+T_{n}^{(3,3)}\right)\left(1+o_{\mathbb{P}}(1)\right)-1\right)(1+o(1)),
$$

where

$$
T_{n}^{(3,2)} \stackrel{\text { def }}{=} \frac{\theta_{n, 1}^{(1)}\left(E_{n-k_{n}+1, n}\right)}{\mu_{1, \tau}\left(E_{n-k_{n}+1, n}\right)}=1+\frac{\sigma_{1, \tau}\left(E_{n-k_{n}+1, n}\right)}{\mu_{1, \tau}\left(E_{n-k_{n}+1, n}\right)} \frac{\xi_{n}^{(1)}}{\sqrt{k_{n}}}=1+o_{\mathbb{P}}(1)
$$




$$
\begin{aligned}
\left|T_{n}^{(3,3)}\right| & \stackrel{\text { def }}{=} \frac{\left|\theta_{n, 3}\left(E_{n-k_{n}+1, n}\right)-\theta_{n, 1}^{(1)}\left(E_{n-k_{n}+1, n}\right)\right|}{\mu_{1, \tau}\left(E_{n-k_{n}+1, n}\right)} \\
& \leq-\frac{\rho}{2} \frac{\theta_{n, 1}^{(2)}\left(E_{n-k_{n}+1, n}\right)}{\mu_{2, \tau}\left(E_{n-k_{n}+1, n}\right)} \frac{\mu_{2, \tau}\left(E_{n-k_{n}+1, n}\right)}{\mu_{1, \tau}\left(E_{n-k_{n}+1, n}\right)} \\
& \stackrel{d}{=}-\rho\left(\log \left(n / k_{n}\right)\right)^{\tau-1}\left(1+o_{\mathbb{P}}(1)\right)\left(1+\frac{\sigma_{2, \tau}\left(E_{n-k_{n}+1, n}\right)}{\mu_{2, \tau}\left(E_{n-k_{n}+1, n}\right)} \frac{\xi_{n}^{(2)}}{\sqrt{k_{n}}}\right) \\
& =O_{\mathbb{P}}\left(\log \left(n / k_{n}\right)\right)^{\tau-1},
\end{aligned}
$$

in view of Lemma 2, Lemma 5 and Lemma 6. Thus, for all $\tau \in[0,1), T_{n}^{(3)} \stackrel{P}{\longrightarrow} 0$. Taking (17) and (18) into account concludes the proof.

Proof of Proposition 4 - From (8), we have

$$
\frac{1}{2} \log k_{n}+\log \left|b\left(\exp K_{\tau}\left(\log \left(n / k_{n}\right)\right)\right)\right| \rightarrow \log |\lambda|
$$

as $n \rightarrow \infty$, and since $K_{\tau}\left(\log \left(n / k_{n}\right)\right) \rightarrow \infty$ as $n \rightarrow \infty$, it follows that

$$
\frac{\log k_{n}}{2 K_{\tau}\left(\log \left(n / k_{n}\right)\right)}+\frac{\log \left|b\left(\exp K_{\tau}\left(\log \left(n / k_{n}\right)\right)\right)\right|}{K_{\tau}\left(\log \left(n / k_{n}\right)\right)} \rightarrow 0
$$

as $n \rightarrow \infty$. Now, $|b|$ is a regularly-varying function with index $\rho$ and thus $\log |b(x)| / \log x \rightarrow \rho$ for all $x \rightarrow \infty$, see [9], Proposition 1.3.6. As a consequence, we obtain

$$
\frac{\log k_{n}}{K_{\tau}\left(\log \left(n / k_{n}\right)\right)} \rightarrow-2 \rho
$$

as $n \rightarrow \infty$. Let us first remark that, if $\tau=1$ then (20) implies

$$
\log k_{n}=\frac{2 \rho}{2 \rho-1}(\log n)(1+o(1))=\frac{2 \rho}{2 \rho-1} K_{1}(\log n)(1+o(1))
$$

and the conclusion follows. Otherwise, if $\tau \in[0,1)$, condition (20) can be rewritten as

$$
\frac{\log k_{n}}{\log n} \frac{\log n}{K_{\tau}\left(\log \left(n / k_{n}\right)\right)} \rightarrow-2 \rho .
$$

Besides, since $K_{\tau}$ is non-decreasing,

$$
\frac{\log n}{K_{\tau}\left(\log \left(n / k_{n}\right)\right)} \geq \frac{\log n}{K_{\tau}(\log n)} \rightarrow \infty
$$

for all $\tau \in[0,1)$ and thus, in view of (21), necessarily $\log k_{n} / \log n \rightarrow 0$ as $n \rightarrow \infty$. As a consequence, $\log \left(n / k_{n}\right)$ is asymptotically equivalent to $\log n$ and thus $K_{\tau}\left(\log \left(n / k_{n}\right)\right)$ is asymptotically equivalent to $K_{\tau}(\log (n))$ as well. Replacing in $(20)$, the conclusion follows.

Proof of Proposition 5 - Let us consider $\tau \in[0,1 / 2)$ and suppose that (8) holds with $\lambda \neq 0$. Following Proposition $4, \log \left(k_{n}\right)=-2 \rho K_{\tau}(\log n)(1+o(1))$ and thus $\log \left(k_{n}\right) / \log (n) \rightarrow 0$ as $n \rightarrow \infty$. A first order Taylor expansion shows that there exists $\eta_{n} \in[0,1]$ such that

$$
\begin{aligned}
\Delta_{n} \stackrel{\text { def }}{=} \exp \left\{K_{\tau}\left(\log \left(n / k_{n}\right)\right)-K_{\tau}(\log n)\right\} & =\exp \left\{-\left(\log k_{n}\right) K_{\tau}^{\prime}\left(\log (n)-\eta_{n} \log \left(k_{n}\right)\right)\right\} \\
& =\exp \left\{-\left(\log k_{n}\right) K_{\tau}^{\prime}(\log n)(1+o(1))\right\},
\end{aligned}
$$


since $K_{\tau}^{\prime}$ is regularly-varying. As a consequence,

$$
\Delta_{n}=\exp \left\{2 \rho K_{\tau}(\log n) K_{\tau}^{\prime}(\log n)(1+o(1))\right\}
$$

and thus $\Delta_{n} \rightarrow 1$ if $\tau \in[0,1 / 2)$ or $\Delta_{n} \rightarrow \exp (4 \rho)$ if $\tau=1 / 2$. Since $b^{2}$ is regularly varying with index $2 \rho$ it follows that

$$
\begin{aligned}
\mathcal{A S B}(\tau, \rho) & =b^{2}\left(\exp K_{\tau}(\log n)\right) \frac{b^{2}\left(\Delta_{n} \exp K_{\tau}(\log n)\right)}{b^{2}\left(\exp K_{\tau}(\log n)\right)} \\
& =c_{\tau, \rho} b^{2}\left(\exp K_{\tau}(\log n)\right)(1+o(1)),
\end{aligned}
$$

and the conclusion follows.

Proof of Theorem 2 - From (7), one can infer that

$$
\begin{aligned}
& \log \widehat{x}_{p_{n}}-\log x_{p_{n}}=\left(\log \left(X_{n-k_{n}+1, n}\right)-\log \bar{F}^{\leftarrow}\left(k_{n} / n\right)\right) \\
&+\left(\widehat{\theta}_{n}\left(k_{n}\right)-\theta\right)\left(K_{\tau}\left(\log \left(1 / p_{n}\right)\right)-K_{\tau}\left(\log \left(n / k_{n}\right)\right)\right) \\
&+ \log \frac{\ell\left(\exp K_{\tau}\left(\log \left(n / k_{n}\right)\right)\right)}{\ell\left(\exp K_{\tau}\left(\log \left(1 / p_{n}\right)\right)\right)} \\
& \stackrel{\text { def }}{=} Q_{n}^{(1)}+Q_{n}^{(2)}+Q_{n}^{(3)} .
\end{aligned}
$$

The three terms are studied separately. First, note that in view of $\left(\mathbf{A}_{1}(\tau, \theta)\right)$ and $\left(\mathbf{A}_{2}(\rho)\right), Q_{n}^{(1)}$ can be expanded as

$$
\begin{aligned}
Q_{n}^{(1)} & =\log H^{\leftarrow}\left(\exp K_{\tau}\left(E_{n-k_{n}+1, n}\right)\right)-\log H^{\leftarrow}\left(\exp K_{\tau}\left(\log \left(n / k_{n}\right)\right)\right) \\
& =\theta\left(K_{\tau}\left(E_{n-k_{n}+1, n}\right)-K_{\tau}\left(\log \left(n / k_{n}\right)\right)\right)+\log \frac{\ell\left(\exp K_{\tau}\left(E_{n-k_{n}+1, n}\right)\right)}{\ell\left(\exp K_{\tau}\left(\log \left(n / k_{n}\right)\right)\right)} \\
& \stackrel{\text { def }}{=} \theta T_{n}^{(3,1)}+Q_{n}^{(1,2)},
\end{aligned}
$$

where $T_{n}^{(3,1)}$ is defined in the proof of Theorem 1 as

$$
T_{n}^{(3,1)}=K_{\tau}\left(E_{n-k_{n}+1, n}\right)-K_{\tau}\left(\log \left(n / k_{n}\right)\right)=O_{\mathbb{P}}(1) \frac{\left(\log \left(n / k_{n}\right)\right)^{\tau-1}}{\sqrt{k_{n}}},
$$

in view of (19). Moreover, $Q_{n}^{(1,2)} \stackrel{\text { def }}{=} \log \ell\left(\lambda_{n} x_{n}\right)-\log \ell\left(x_{n}\right)$, where $x_{n}=\exp K_{\tau}\left(\log \left(n / k_{n}\right)\right) \rightarrow \infty$ and $\lambda_{n}=\exp T_{n}^{(3,1)} \stackrel{P}{\longrightarrow} 1$. Thus, from $\left(\mathbf{A}_{2}(\rho)\right)$ we have

$$
\begin{aligned}
Q_{n}^{(1,2)} & =b\left(\exp K_{\tau}\left(\log \left(n / k_{n}\right)\right)\right) K_{\rho}\left(\lambda_{n}\right)\left(1+o_{\mathbb{P}}(1)\right) \\
& =b\left(\exp K_{\tau}\left(\log \left(n / k_{n}\right)\right)\right) \log \left(\lambda_{n}\right)\left(1+o_{\mathbb{P}}(1)\right) \\
& =O_{\mathbb{P}}(1) b\left(\exp K_{\tau}\left(\log \left(n / k_{n}\right)\right)\right) \frac{\left(\log \left(n / k_{n}\right)\right)^{\tau-1}}{\sqrt{k_{n}}},
\end{aligned}
$$

in view of $(22)$. Since $b(x) \rightarrow 0$ as $x \rightarrow \infty$, it follows that

$$
Q_{n}^{(1,2)}=o_{\mathbb{P}}\left(\frac{\left(\log \left(n / k_{n}\right)\right)^{\tau-1}}{\sqrt{k_{n}}}\right)
$$

entailing

$$
\frac{\sqrt{k_{n}}}{K_{\tau}\left(\log \left(1 / p_{n}\right)\right)-K_{\tau}\left(\log \left(n / k_{n}\right)\right)} Q_{n}^{(1)}=O_{\mathbb{P}}\left(\frac{\left(\log \left(n / k_{n}\right)\right)^{\tau-1}}{K_{\tau}\left(\log \left(1 / p_{n}\right)\right)-K_{\tau}\left(\log \left(n / k_{n}\right)\right)}\right)=o_{\mathbb{P}}(1),
$$


from (10). Now, concerning the second term, Theorem 1 entails that

$$
\frac{\sqrt{k_{n}}}{K_{\tau}\left(\log \left(1 / p_{n}\right)\right)-K_{\tau}\left(\log \left(n / k_{n}\right)\right)} Q_{n}^{(2)}=\sqrt{k_{n}}\left(\widehat{\theta}_{n}\left(k_{n}\right)-\theta\right) \stackrel{d}{\longrightarrow} \mathcal{N}\left(0, \theta^{2}\right) .
$$

Finally, $Q_{n}^{(3)}=\log \ell\left(x_{n}^{*}\right)-\log \ell\left(\lambda_{n}^{*} x_{n}^{*}\right)$ where $\lambda_{n}^{*}=\exp \left[K_{\tau}\left(\log \left(1 / p_{n}\right)\right)-K_{\tau}\left(\log \left(n / k_{n}\right)\right)\right] \geq 1$ in view of (10) and $x_{n}^{*}=\exp K_{\tau}\left(\log \left(n / k_{n}\right)\right) \rightarrow \infty$. Thus, Lemma 1 entails

$$
\frac{\sqrt{k_{n}} Q_{n}^{(3)}}{\log \lambda_{n}^{*}} \sim-\sqrt{k_{n}} b\left(x_{n}^{*}\right) \frac{K_{\rho}\left(\lambda_{n}^{*}\right)}{\log \lambda_{n}^{*}}=o\left(\frac{K_{\rho}\left(\lambda_{n}^{*}\right)}{\log \lambda_{n}^{*}}\right),
$$

since $\sqrt{k_{n}} b\left(x_{n}^{*}\right)=\sqrt{k_{n}} b\left(\exp K_{\tau}\left(\log \left(n / k_{n}\right)\right)\right) \rightarrow 0$. Taking account of the inequality $K_{\rho}(x) \leq \log x$ for all $x \geq 1$ yields

$$
\frac{\sqrt{k_{n}}}{K_{\tau}\left(\log \left(1 / p_{n}\right)\right)-K_{\tau}\left(\log \left(n / k_{n}\right)\right)} Q_{n}^{(3)}=o(1) .
$$

Combining the above results, Theorem 2 follows.

\section{Acknowledgments}

The authors are grateful to the referees for their helpful comments and to Jonathan El Methni for suggesting the estimator of $\tau$.

\section{References}

[1] Arnold, B.C, Balakrishnan, N., Nagaraja H.N., (1992), A First course in order statistics, Wiley and sons.

[2] Asimit, V., Li, D., Peng, L., (2010), Pitfalls in using Weibull tailed distributions, Journal of Statistical Planning and Inference 140, 2018-2024.

[3] Beirlant, J., Bouquiaux, C., Werker, B., (2006), Semiparametric lower bounds for tail index estimation, Journal of Statistical Planning and Inference, 136, 705-729.

[4] Beirlant, J., Broniatowski, M., Teugels, J.L., Vynckier, P., (1995), The mean residual life function at great age: Applications to tail estimation, Journal of Statistical Planning and Inference, 45, 21-48.

[5] Beirlant, J., Dierckx, G., Goegebeur, Y., Matthys, G., (1999), Tail index estimation and an exponential regression model, Extremes, 2, 177-200.

[6] Beirlant, J., Teugels, J.L., (1992), Modeling large claims in non-life insurance. Insurance: Mathematics and Economics, 11, 17-29.

[7] Beirlant, J., Teugels, J., Vynckier, P., (1996), Practical analysis of extreme values, Leuven university press, Leuven.

[8] Berred, M., (1991), Record values and the estimation of the Weibull tail-coefficient, Comptes Rendus de l'Académie des Sciences, T. 312, Série I, 943-946.

[9] Bingham, N.H., Goldie, C.M., Teugels, J.L., (1987), Regular Variation, Cambridge University Press. 
[10] Broniatowski, M., (1993), On the estimation of the Weibull tail coefficient, Journal of Statistical Planning and Inference, 35, 349-366.

[11] Diebolt, J., Gardes, L., Girard, S., Guillou, A., (2008a), Bias-reduced estimators of the Weibull tail-coefficient, Test, 17, 311-331.

[12] Diebolt, J., Gardes, L., Girard, S., Guillou, A., (2008b), Bias-reduced extreme quantiles estimators of Weibull tail-distributions, Journal of Statistical Planning and Inference, 138, $1389-1401$.

[13] Dierckx, G., Beirlant, J., De Waal, D., Guillou, A. (2009), A new estimation method for Weibull-type tails based on the mean excess function, Journal of the Statistical Planning and Inference, 139, 1905-1920.

[14] Ditlevsen, O., (1994), Distribution Arbitrariness in Structural Reliability, Structural Safety and Reliability, 1241-1247, Balkema, Rotterdam.

[15] Draisma, G., de Haan, L., Peng, L., Peireira, T. T., (1999), A Bootstrap-based method to achieve optimality in estimating the extreme-value index, Extremes, 2:4, 367-404.

[16] Embrechts, P., Klüppelberg, C., Mikosch, T., (1997), Modelling extremal events, Springer.

[17] Falk, M., (1995), Some best parameter estimates for distributions with finite endpoint, Statistics, 27, 115-125.

[18] Feuerverger, A., Hall, P., (1999), Estimating a tail exponent by modelling departure from a Pareto distribution, Annals of Statistics, 27, 760-781.

[19] Fraga Alves, M.I, de Haan, L., Neves, C., (2009), A test procedure for detecting super heavy tails, Journal of Statistical Planning and Inference, 139, 213-227.

[20] Galambos, J., (1987), The Asymptotic theory of extreme order statistics, R.E. Krieger publishing company.

[21] Gardes, L., Girard, S., (2005), Estimating extreme quantiles of Weibull tail-distributions, Communication in Statistics - Theory and Methods, 34, 1065-1080.

[22] Gardes, L., Girard, S., (2006), Comparison of Weibull tail-coefficient estimators, REVSTAT - Statistical Journal, 4, 163-188.

[23] Gardes, L., Girard, S., (2008), Estimation of the Weibull tail-coefficient with linear combination of upper order statistics, Journal of Statistical Planning and Inference, 138, 1416-1427.

[24] Geluk, J.L., de Haan, L., (1987), Regular Variation, Extensions and Tauberian Theorems, Math Centre Tracts, 40, Centre for Mathematics and Computer Science, Amsterdam.

[25] Girard, S., (2004), A Hill type estimate of the Weibull tail-coefficient, Communication in Statistics - Theory and Methods, 33(2), 205-234.

[26] Goegebeur,Y., Beirlant, J., de Wet, T. (2010), Generalized kernel estimators for the Weibull-tail coefficient, Communications in Statistics - Theory and Methods, to appear, http://bib.mathematics.dk/preprint.php?id=IMADA-PP-2009-09

[27] Goegebeur, Y., Guillou, A. (2010), Goodness-of-fit testing for Weibull-type behavior, Journal of Statistical Planning and Inference, 140, 1417-1436. 
[28] Gomes, I., Caeiro, F., (2002), A class of asymptotically unbiased semi-parametric estimators of the tail index, Test, 11(2), 345-364.

[29] Gomes, I., Caeiro, F., Figueiredo, F., (2004), Bias reduction of a tail index estimator through an external estimation of the second-order parameter, Statistics, 38(6), 497-510.

[30] de Haan, L., Ferreira, A., (2006), Extreme Value Theory: An Introduction, Springer Series in Operations Research and Financial Engineering, Springer.

[31] de Haan, L., Peng, L., (1998), Comparison of tail index estimators, Statistica Neerlandica, 52(1), 60-70.

[32] Harris, R.J., (1996), Gumbel re-visited - a new look at extreme value statistics applied to wind speeds, Journal of Wind Engineering and Industrial Aerodynamics, 59, 1-22.

[33] Häusler, E., Teugels, J.L. (1985), On asymptotic normality of Hill's estimator for the exponent of regular variation, The Annals of Statistics, 13, 743-756.

[34] Hill, B.M., (1975), A simple general approach to inference about the tail of a distribution, The Annals of Statistics, 3, 1163-1174.

[35] Petrov, V.V., (1975), Sums of independent random variables, Springer-Verlag, Berlin, Eidelberg, New York.

[36] Smith, J., (1991), Estimating the upper tail of flood frequency distributions, Water Resources Research, 23(8), 1657-1666.

[37] Weissman, I., (1978), Estimation of parameters and large quantiles based on the $k$ largest observations, Journal of the American Statistical Association, 73, 812-815.

\section{Appendix: Proof of auxiliary results}

Proof of Lemma $\mathbf{1}$ - From $\left(\mathbf{A}_{1}(\tau, \theta)\right)$ and $\left(\mathbf{A}_{2}(\rho)\right)$, it is easy to infer that, for any constant $\widetilde{C}>0$, we have

$$
\begin{aligned}
\frac{1}{\widetilde{C} b(x)}\left(\frac{H^{\leftarrow}(\lambda x)-H^{\leftarrow}(x)}{\theta H^{\leftarrow}(x)(1+b(x) / \theta)}-\frac{\lambda^{\theta}-1}{\theta}\right) & =\frac{\lambda^{\theta}}{\widetilde{C} \theta} K_{\rho}(\lambda)-\frac{1}{\widetilde{C} \theta} \frac{\lambda^{\theta}-1}{\theta}+o(1) \\
& =\frac{\theta+\rho}{\widetilde{C} \theta} \frac{1}{\rho}\left[K_{\theta+\rho}(\lambda)-K_{\theta}(\lambda)\right]+o(1) .
\end{aligned}
$$

Then, choosing $\widetilde{C}$ such that $(\theta+\rho) /(\widetilde{C} \theta)=1$, a direct application of Lemma 5.2 in [15] yields, for any $\varepsilon>0$ and $\lambda \geq 1$,

$$
\begin{aligned}
& \min \left(1, \lambda^{-\rho-\varepsilon}\right)\left|\frac{\ell(\lambda x)}{\ell(x)}-1-b(x) K_{\rho}(\lambda)-\frac{1}{\theta} b^{2}(x)\left[K_{\rho}(\lambda)-K_{-\theta}(\lambda)\right]\right| \\
\leq & \varepsilon \widetilde{C} \theta|b(x)||1+b(x) / \theta| \min \left(1, \lambda^{-\rho-\varepsilon}\right)\left[\lambda^{-\theta}+1+2 \lambda^{\rho+\varepsilon}\right] \\
\leq & 4 \varepsilon \widetilde{C} \theta|b(x)| \min \left(1, \lambda^{-\rho-\varepsilon}\right)\left[1+\lambda^{\rho+\varepsilon}\right] \\
\leq & 8 \varepsilon \widetilde{C} \theta|b(x)|
\end{aligned}
$$

for $x$ large enough. Moreover, letting $0<\varepsilon<-\rho$ yields

$$
\sup _{\lambda \geq 1}\left|\frac{\ell(\lambda x)}{\ell(x)}-1-b(x) K_{\rho}(\lambda)-\frac{1}{\theta} b^{2}(x)\left[K_{\rho}(\lambda)-K_{-\theta}(\lambda)\right]\right|=o(b(x)) .
$$


Besides, $K_{\rho}(\lambda)-K_{-\theta}(\lambda)$ is bounded when $\rho<0$, and therefore (23) can be simplified as

$$
\sup _{\lambda \geq 1}\left|\frac{\ell(\lambda x)}{\ell(x)}-1-b(x) K_{\rho}(\lambda)\right|=o(b(x)), \text { as } x \rightarrow \infty,
$$

and the conclusion follows.

Proof of Lemma 2 - (i) Let us consider for $t>1$ and $q \in \mathbb{N} \backslash\{0\}$,

$$
Q_{q}(t)=\int_{0}^{\infty}\left(\frac{K_{\tau}(x+t)-K_{\tau}(t)}{K_{\tau}^{\prime}(t)}\right)^{q} \mathrm{e}^{-x} d x
$$

There exists $\eta \in(0,1)$ such that

$$
\left|\frac{K_{\tau}(x+t)-K_{\tau}(t)}{x K_{\tau}^{\prime}(t)}\right|=\left(1+\frac{\eta x}{t}\right)^{\tau-1} \leq 1
$$

Thus, Lebesgue Theorem implies that

$$
\lim _{t \rightarrow \infty} Q_{q}(t)=\int_{0}^{\infty} \lim _{t \rightarrow \infty}\left(1+\frac{\eta x}{t}\right)^{q(\tau-1)} x^{q} \mathrm{e}^{-x} d x=\int_{0}^{\infty} x^{q} \mathrm{e}^{-x} d x=q !
$$

which concludes the first part of the proof.

(ii) is a straightforward consequence of (i).

(iii) We have

$$
\begin{aligned}
\mu_{1, \tau}^{\prime}(t) & =\int_{0}^{\infty}\left(K_{\tau}^{\prime}(x+t)-K_{\tau}^{\prime}(t)\right) \mathrm{e}^{-x} d x \\
& =\int_{0}^{\infty} K_{\tau}^{\prime}(x+t) \mathrm{e}^{-x} d x-K_{\tau}^{\prime}(t) \\
& =\int_{0}^{\infty} K_{\tau}(x+t) \mathrm{e}^{-x} d x-K_{\tau}(t)-K_{\tau}^{\prime}(t) \\
& =\mu_{1, \tau}(t)-t^{\tau-1} .
\end{aligned}
$$

Finally, (i) states that $t^{\tau-1} / \mu_{1, \tau}(t) \rightarrow 1$ as $t \rightarrow \infty$ which entails $\mu_{1, \tau}^{\prime}(t) / \mu_{1, \tau}(t) \rightarrow 0$ as $t \rightarrow \infty$.

Proof of Lemma 3 - Recall that

$$
\begin{aligned}
\widehat{\theta}_{n} & =\frac{1}{\mu_{1, \tau}\left(\log \left(n / k_{n}\right)\right)} \frac{1}{k_{n}-1} \sum_{i=1}^{k_{n}-1}\left(\log \left(X_{n-i+1, n}\right)-\log \left(X_{n-k_{n}+1, n}\right)\right) \\
& \stackrel{d}{=} \frac{1}{\mu_{1, \tau}\left(\log \left(n / k_{n}\right)\right)} \frac{1}{k_{n}-1} \sum_{i=1}^{k_{n}-1} \log \left(\frac{H^{\leftarrow}\left(\exp K_{\tau}\left(E_{n-i+1, n}\right)\right)}{H^{\leftarrow}\left(\exp K_{\tau}\left(E_{n-k_{n}+1, n}\right)\right)}\right),
\end{aligned}
$$

where $E_{1, n}, \ldots, E_{n, n}$ are ordered statistics generated by $n$ independent standard exponential random variables. The Rényi representation of the $\operatorname{Exp}(1)$ ordered statistics (see [1], p. 72) yields

$$
\left\{E_{n-i+1, n}\right\}_{i=1, \ldots, k_{n}-1} \stackrel{d}{=}\left\{F_{k_{n}-i, k_{n}-1}+E_{n-k_{n}+1, n}\right\}_{i=1, \ldots, k_{n}-1},
$$

where $\left\{F_{1, k_{n}-1}, \ldots, F_{k_{n}-1, k_{n}-1}\right\}$ are ordered statistics independent from $E_{n-k_{n}+1, n}$ and generated by $k_{n}-1$ independent standard exponential variables $\left\{F_{1}, \ldots, F_{k_{n}-1}\right\}$. We thus have

$$
\widehat{\theta}_{n}\left(k_{n}\right) \stackrel{d}{=} \frac{1}{\mu_{1, \tau}\left(\log \left(n / k_{n}\right)\right)} \frac{1}{k_{n}-1} \sum_{i=1}^{k_{n}-1} \log \left(\frac{H^{\leftarrow}\left(\exp K_{\tau}\left(F_{k_{n}-i, k_{n}-1}+E_{n-k_{n}+1, n}\right)\right)}{\left.H^{\leftarrow}\left(\exp K_{\tau}\left(E_{n-k_{n}+1, n}\right)\right)\right)}\right)
$$




$$
\begin{aligned}
& \stackrel{d}{=} \frac{1}{\mu_{1, \tau}\left(\log \left(n / k_{n}\right)\right)} \frac{1}{k_{n}-1} \sum_{i=1}^{k_{n}-1} \log \left(\frac{H^{\leftarrow}\left(\exp K_{\tau}\left(F_{i}+E_{n-k_{n}+1, n}\right)\right)}{H^{\leftarrow}\left(\exp K_{\tau}\left(E_{n-k_{n}+1, n}\right)\right)}\right) \\
& \stackrel{d}{=} \frac{1}{\mu_{1, \tau}\left(\log \left(n / k_{n}\right)\right)}\left(\theta \theta_{n, 1}^{(1)}\left(E_{n-k_{n}+1, n}\right)+\theta_{n, 2}\left(E_{n-k_{n}+1, n}\right)\right)
\end{aligned}
$$

in view of $\left(\mathbf{A}_{1}(\tau, \theta)\right)$ and the conclusion follows.

Proof of Lemma 4 - A first order expansion of the function $g$ leads to,

$$
\left.\sqrt{k_{n}}\left(g\left(E_{n-k_{n}+1, n}\right)-g\left(\log n / k_{n}\right)\right)\right)=\sqrt{k_{n}}\left(E_{n-k_{n}+1, n}-\log \left(n / k_{n}\right)\right) g^{\prime}\left(\tilde{\eta}_{n}\right),
$$

with $\tilde{\eta}_{n} \in\left[\min \left(E_{n-k_{n}+1, n}, \log \left(n / k_{n}\right)\right), \max \left(E_{n-k_{n}+1, n}, \log \left(n / k_{n}\right)\right)\right]$. Now, Lemma 1 in [25] shows that $\sqrt{k_{n}}\left(E_{n-k_{n}+1, n}-\log \left(n / k_{n}\right)\right) \stackrel{d}{\longrightarrow} \mathcal{N}(0,1)$ which implies that $\tilde{\eta}_{n}=\log \left(n / k_{n}\right)\left(1+o_{\mathbb{P}}(1)\right)$ and the result follows.

Proof of Lemma 5 - Let us introduce for all $t \geq 1$ and $q \in \mathbb{N} \backslash\{0\}$,

$$
S_{n}^{(q)}(t)=\frac{\left(k_{n}-1\right)^{1 / 2}}{\sigma_{q, \tau}(t)}\left(\theta_{n, 1}^{(q)}(t)-\mu_{q, \tau}(t)\right)=\frac{\left(k_{n}-1\right)^{-1 / 2}}{\sigma_{q, \tau}(t)} \sum_{i=1}^{k_{n}-1} Y_{i}^{(q)}(t),
$$

where $Y_{i}^{(q)}(t) \stackrel{\text { def }}{=}\left(K_{\tau}\left(F_{i}+t\right)-K_{\tau}(t)\right)^{q}-\mu_{q, \tau}(t), i=1, \ldots, k_{n}-1$ are centered, independent and identically distributed random variables with variance $\sigma_{q, \tau}^{2}(t)$. Clearly, in view of the Central Limit Theorem, for all $t \geq 1$ and $q \in \mathbb{N} \backslash\{0\}, S_{n}^{(q)}(t)$ converges in distribution to a standard Gaussian distribution. Our goal is to prove that, for all $x \in \mathbb{R}$ and $q \in \mathbb{N} \backslash\{0\}$,

$$
\mathbb{P}\left(S_{n}^{(q)}\left(E_{n-k_{n}+1, n}\right) \leq x\right) \rightarrow \Phi(x) \text { as } n \rightarrow \infty,
$$

where $\Phi$ is the cumulative distribution function of the standard Gaussian distribution. Lemma 2(i) implies that for all $\varepsilon \in(0,1)$, and $r \in \mathbb{N} \backslash\{0\}$, there exists $T_{\varepsilon} \geq 1$ such that for all $t \geq T_{\varepsilon}$,

$$
(1-\varepsilon) r ! t^{r(\tau-1)} \leq \mu_{r, \tau}(t) \leq(1+\varepsilon) r ! t^{r(\tau-1)} .
$$

Furthermore, for $x \in \mathbb{R}$,

$$
\begin{aligned}
\mathbb{P}\left(S_{n}^{(q)}\left(E_{n-k_{n}+1, n}\right) \leq x\right)-\Phi(x) & =\int_{0}^{T_{\varepsilon}}\left(\mathbb{P}\left(S_{n}^{(q)}(t) \leq x\right)-\Phi(x)\right) h_{n}(t) d t \\
& +\int_{T_{\varepsilon}}^{\infty}\left(\mathbb{P}\left(S_{n}^{(q)}(t) \leq x\right)-\Phi(x)\right) h_{n}(t) d t \stackrel{\text { def }}{=} A_{n}+B_{n},
\end{aligned}
$$

where $h_{n}$ is the density of the random variable $E_{n-k_{n}+1, n}$. First, let us focus on the term $A_{n}$. We have,

$$
\left|A_{n}\right| \leq 2 \mathbb{P}\left(E_{n-k_{n}+1, n} \leq T_{\varepsilon}\right) .
$$

Since $E_{n-k_{n}+1, n} / \log (n / k) \stackrel{P}{\longrightarrow} 1$ (see [25], Lemma 1), it is easy to show that $A_{n} \rightarrow 0$. Now, let us consider the term $B_{n}$. For all $t \geq T_{\varepsilon}$,

$$
\begin{aligned}
\mathbb{E}\left(\left|Y_{1}^{(q)}(t)\right|^{3}\right) & \leq \mathbb{E}\left(\left(K_{\tau}\left(F_{1}+t\right)-K_{\tau}(t)\right)^{q}+\mu_{q, \tau}(t)\right)^{3} \\
& =\mu_{3 q, \tau}(t)+3 \mu_{q, \tau}(t) \mu_{2 q, \tau}(t)+4 \mu_{q, \tau}^{3}(t) \\
& \leq C_{1}(\varepsilon) t^{3 q(\tau-1)}<\infty
\end{aligned}
$$


from (25). Here, and in the following, $C_{1}(\varepsilon), C_{2}, C_{3}(\varepsilon)$ and $C_{4}(\varepsilon)$ are positive constants independent of $t$. Thus, from Berry-Esséen's inequality (see [35], Theorem 3), we have:

$$
\sup _{x}\left|\mathbb{P}\left(S_{n}^{(q)}(t) \leq x\right)-\Phi(x)\right| \leq C_{2} L_{n} \text { with } L_{n}=\frac{\left(k_{n}-1\right)^{-1 / 2}}{\sigma_{q, \tau}^{3}(t)} \mathbb{E}\left(\left|Y_{1}^{(q)}(t)\right|^{3}\right) .
$$

From (25), since $t \geq T_{\varepsilon}$,

$$
\sigma_{q, \tau}^{2}(t)=\mu_{2 q, \tau}(t)-\mu_{q, \tau}^{2}(t) \geq C_{3}(\varepsilon) t^{2 q(\tau-1)} .
$$

Thus, $L_{n} \leq C_{4}(\varepsilon)\left(k_{n}-1\right)^{-1 / 2}$ and therefore

$$
\left|B_{n}\right| \leq C_{2} C_{4}(\varepsilon)\left(k_{n}-1\right)^{-1 / 2} \mathbb{P}\left(E_{n-k_{n}+1, n} \geq T_{\varepsilon}\right) \leq C_{2} C_{4}(\varepsilon)\left(k_{n}-1\right)^{-1 / 2} \rightarrow 0,
$$

which concludes the proof.

Proof of Lemma 6 - Let us consider the random variables $x_{n}=\exp \left[K_{\tau}\left(E_{n-k_{n}+1, n}\right)\right]$ and $\lambda_{i, n}=\exp \left[K_{\tau}\left(F_{i}+E_{n-k_{n}+1, n}\right)-K_{\tau}\left(E_{n-k_{n}+1, n}\right)\right], i=1, \ldots, k_{n}-1$. It is clear that $x_{n} \stackrel{P}{\longrightarrow} \infty$ in view of Lemma 1 in [25] and $\lambda_{i, n} \geq 1$. Thus, letting

$$
\theta_{n, 3}\left(E_{n-k_{n}+1, n}\right)=\frac{1}{k_{n}-1} \sum_{i=1}^{k_{n}-1} K_{\rho}\left[\exp \left(K_{\tau}\left(F_{i}+E_{n-k_{n}+1, n}\right)-K_{\tau}\left(E_{n-k_{n}+1, n}\right)\right)\right],
$$

Lemma 1 entails

$$
\theta_{n, 2}\left(E_{n-k_{n}+1, n}\right) \stackrel{d}{=} b\left(\exp K_{\tau}\left(E_{n-k_{n}+1, n}\right)\right) \theta_{n, 3}\left(E_{n-k_{n}+1, n}\right)\left(1+o_{\mathbb{P}}(1)\right) .
$$

Since $\left|K_{\rho}(\exp u)-u\right| \leq-\rho u^{2} / 2$ for all $u \geq 0$, we have

$$
\left|\theta_{n, 3}\left(E_{n-k_{n}+1, n}\right)-\theta_{n, 1}^{(1)}\left(E_{n-k_{n}+1, n}\right)\right| \leq-\frac{\rho}{2} \theta_{n, 1}^{(2)}\left(E_{n-k_{n}+1, n}\right) .
$$

Moreover, if $\tau=1$, then

$$
\theta_{n, 3}\left(E_{n-k_{n}+1, n}\right)=\frac{1}{k_{n}-1} \sum_{i=1}^{k_{n}-1} K_{\rho}\left(\exp F_{i}\right) \stackrel{P}{\longrightarrow} \int_{0}^{+\infty} K_{\rho}(\exp u) \exp (-u) d u=\frac{1}{1-\rho},
$$

in view of the law of large numbers, and the conclusion follows. 

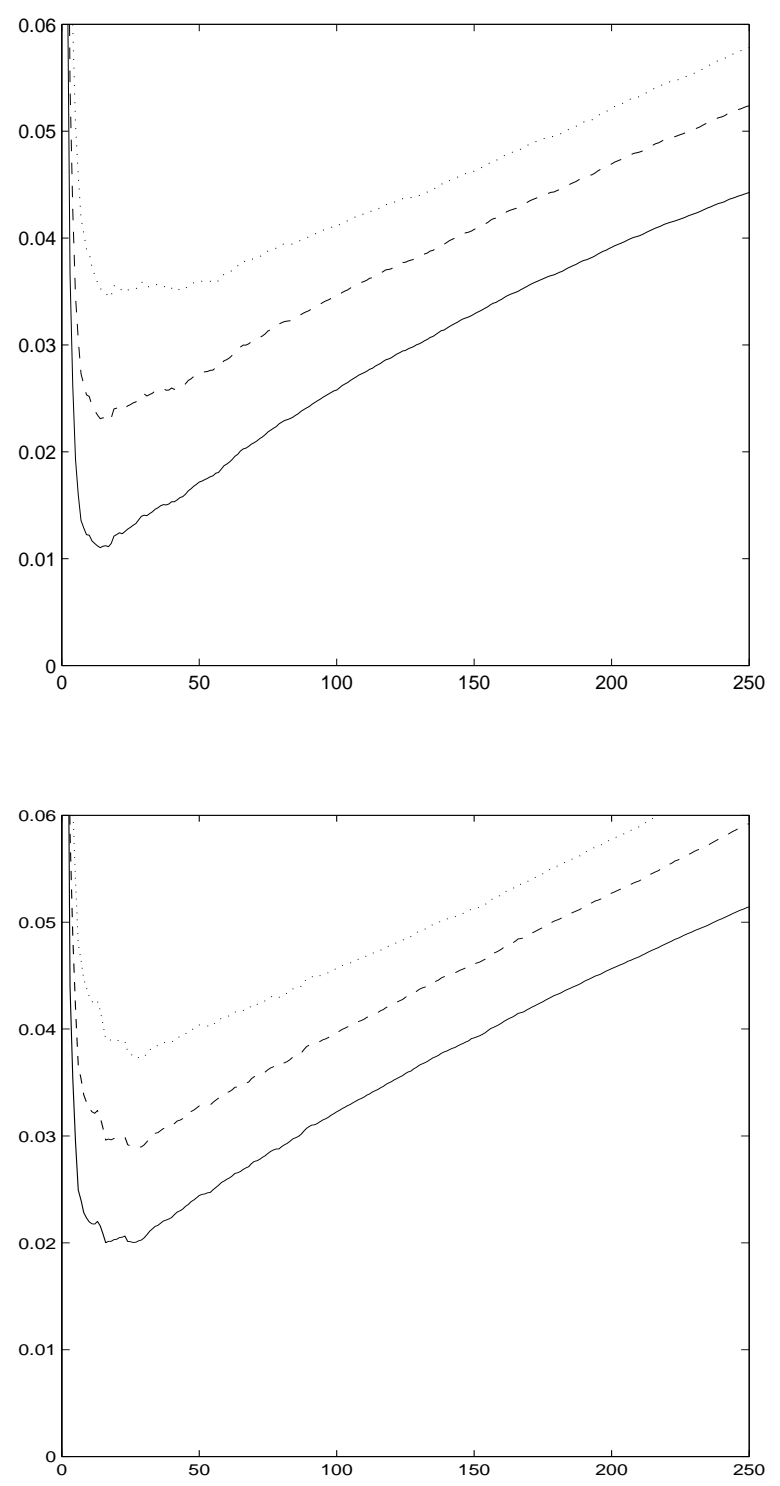

Figure 1: Empirical squared bias as a function of $k$ obtained with $\widehat{\theta}_{n}\left(k_{n}\right)$ computed on 500 samples of size 500 from $F_{1 / 2, \tau, \rho}$. Up: $\rho=-1 / 2$, down: $\rho=-1 / 4$, solid line: $\tau=1$, dashed line: $\tau=1 / 2$, dotted line: $\tau=0$. 

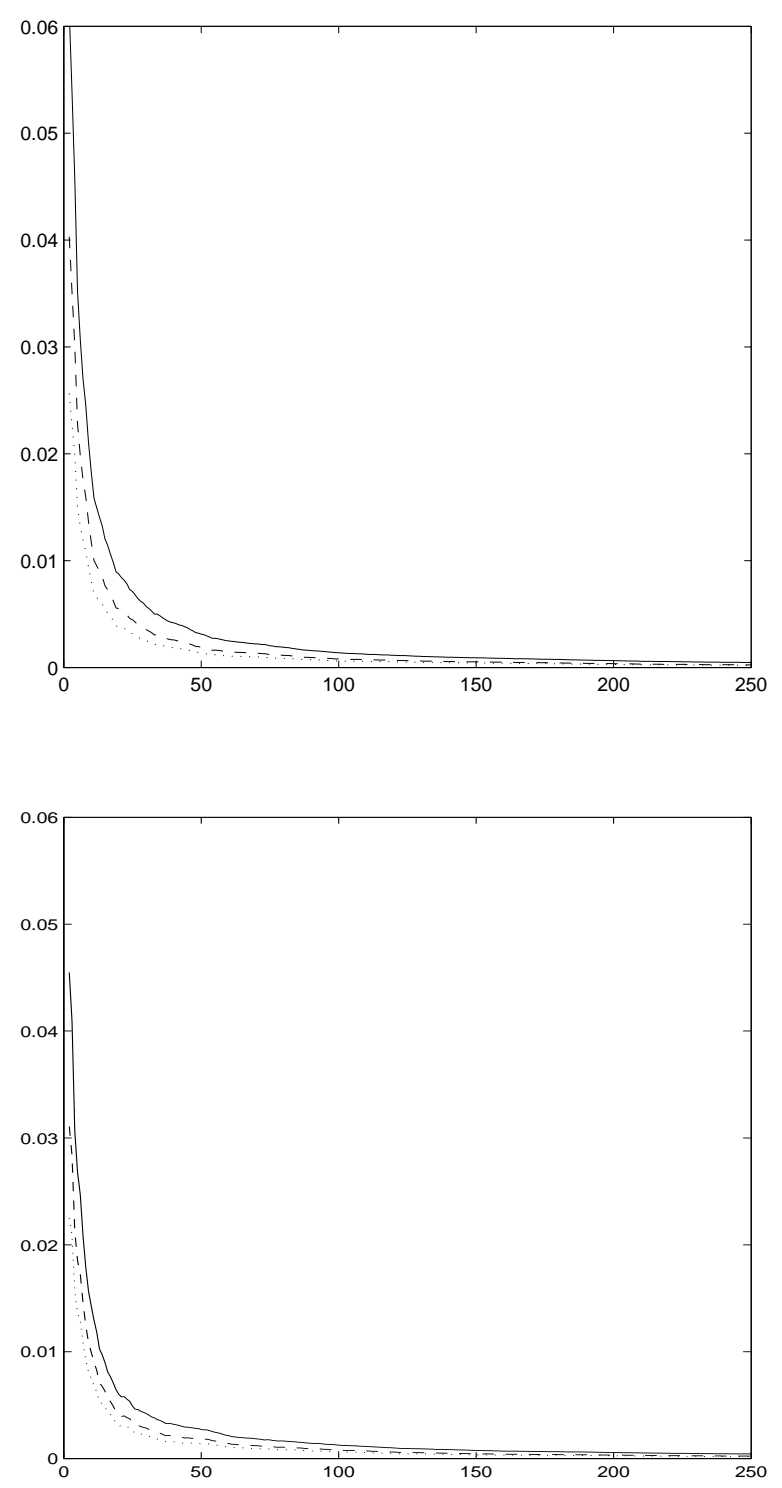

Figure 2: Empirical variance as a function of $k$ obtained with $\widehat{\theta}_{n}\left(k_{n}\right)$ computed on 500 samples of size 500 from $F_{1 / 2, \tau, \rho}$. Up: $\rho=-1 / 2$, down: $\rho=-1 / 4$, solid line: $\tau=1$, dashed line: $\tau=1 / 2$, dotted line: $\tau=0$. 